\title{
Improving phase congruency for EEG data reduction
}

\author{
Lojini Logesparan and Esther Rodriguez-Villegas
}

\begin{abstract}
Real signals are often corrupted by noise. In applications where the noise power spectrum is variable with time, dynamic noise estimation and compensation can potentially improve the performance of signal processing algorithms. One such application is scalp EEG monitoring in epilepsy, where the electrical activity generated by cranio-facial muscle contraction and expansion, often obscures the measured brainwave signals. This work presents a data reduction algorithm which is based on differentiating interictal from normal background activity, in epileptic scalp EEG signals, using a modified phase congruency technique. The modification is based on dynamically estimating muscle activity from the signal and incorporating this estimation in phase congruency computations. The proposed algorithm identifies $90 \%$ of interictal spikes whilst transmitting only $45 \%$ of EEG data. This is in the order of $15 \%$ improvement in data reduction when compared to the performance obtained with the state-of-the-art denoised phase congruency-which calculates a constant noise threshold-applied to the same dataset.
\end{abstract}

\section{INTRODUCTION}

Epilepsy is a common neurological disorder that affects 50 million people worldwide [1]. Electroencephalography (EEG) is a key tool for diagnosing the condition. The benefits of long term ambulatory EEG monitoring for both diagnosis and treatment have been extensively reported [1][3]. Unfortunately, the lack of in-patient resources coupled with limitations of current EEG technologies for out-patient monitoring make long term monitoring an option to only those with refractory epilepsy [1]. During an ambulatory test, the patient is tethered to a portable EEG system for a period that can vary from hours to even weeks. Ambulatory EEG systems (AEEG) are battery operated but despite this, current battery packs are still too bulky and heavy which significantly limits the patients' ability to carry out normal activities [4]. From the usability point of view, the limitations of current AEEG technologies could potentially be overcome by a miniaturized solution, in which the signals are wireless transmitted to a receiver. However, this poses an important technological problem: wireless transmission is power hungry [4]. It also determines the size and duration of the battery. Power consumption could be reduced by either fully customizing the design of all the electronic blocks that form the EEG system [5] and/or coming up with strategies to reduce the amount of data that needs to be transmitted.

This paper presents a data selection algorithm to identify candidate epileptic interictal activity and reject normal back-

L. Logesparan and E. Rodriguez-Villegas are with the Electrical and Electronic Engineering Department, Imperial College London, SW7 2AZ \{lojini.logesparan04, e.rodriguez\}@imperial.ac.uk

The research leading to these results has received funding from the European Research Council under the European Community's 7th Framework Programme (FP7/2007-2013) / ERC grant agreement no. 239749 ground brain activity. This retains the necessary information for diagnostic purposes whilst reducing the amount of data that would need to be transmitted. The proposed algorithm bases its performance on the relationship between the phase of different frequency components of the signal at any instant in time, since it is found that the latter changes considerably between background and interictal activity. Analyzing the relationship between the different phases of a signal at a certain point in time is a technique that has been widely used in image processing as a tool for detecting edges, steps and impulses [6]-[8], and is generally known as phase congruency. Originally phase congruency was calculated just using the local energy function and amplitudes of multiple frequency components of the input signal [9]. This method was later modified to include an estimation of noise. The most cited method to remove noise [6] assumes a constant noise power spectrum to estimate a fixed noise threshold, which is incorporated in the phase congruency calculation. However, many real signals (including EEG) do not have a constant noise power spectrum. The algorithm proposed in this paper is based on a novel modification to the phase congruency technique, tailored to scalp EEG signals. This modification is based on dynamically estimating the interference generated by the cranio-facial muscles and incorporating this estimation into the phase congruency calculations.

The paper is organized as follows: Section II describes the traditional phase congruency calculation, the modification to phase congruency by estimating muscle artefacts and the data selection algorithm for identifying interictal spikes. The performance of the algorithm is analyzed in Section III and compared with the performance of traditional phase congruency and denoised phase congruency [6] on the same test database.

\section{Methods}

The proposed algorithm computes modified phase congruency as the feature for identifying candidate spikes and rejecting background activity. An interictal spike is a sharp transient with a duration ranging from less than $70 \mathrm{~ms}$ up to $200 \mathrm{~ms}$ [10]. Hence, it is expected that a transient would have higher phase congruency than background activity. This difference in phase congruency between spikes and background is exploited in the algorithm to extract candidate spikes.

\section{A. Phase congruency}

Phase information at different frequencies can be extracted using log Gabor wavelet filters [6]. Log Gabor filters are better suited for this application because they provide zero dc component at wide bandwidth. To avoid phase distortion by the filters, a linear phase response is designed 


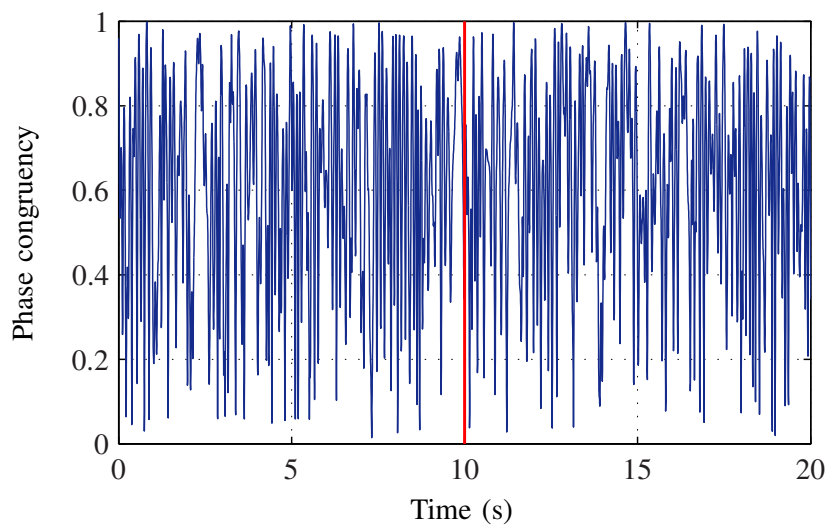

(a) Phase congruency

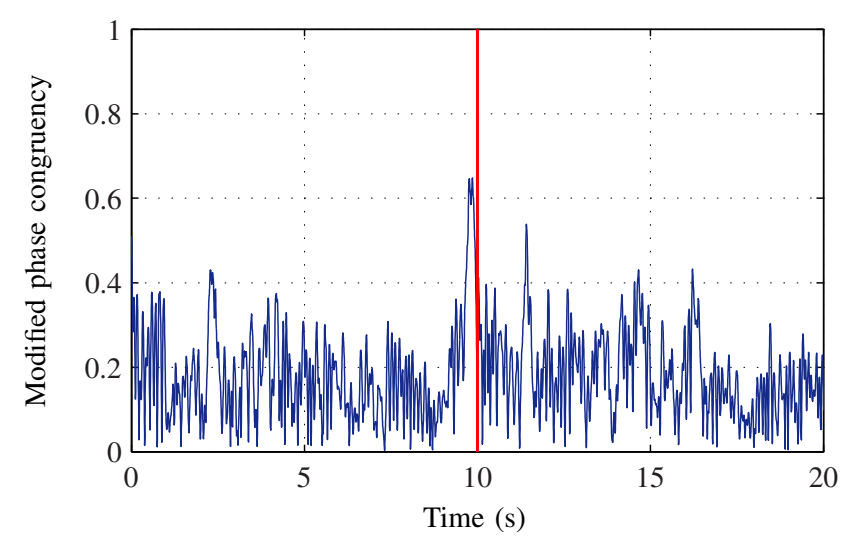

(b) Modified phase congruency

Fig. 1. Phase congruency and modified phase congruency calculated for $20 \mathrm{~s}$ EEG data with a single expert marked spike at $10 \mathrm{~s}$

using nonorthogonal wavelets in symmetric/anti-symmetric quadrature pairs [6].

Considering an input EEG signal $I(x)$ at a time $x$, the even-symmetric and odd-symmetric filter components are $M_{n}^{e}$ and $M_{n}^{o}$ respectively, where $n$ represents the wavelet scale which relates to the frequency bands of the filters [6]. The output even and odd-symmetric wavelet coefficients are defined as:

$$
\left[e_{n}(x), o_{n}(x)\right]=\left[I(x) * M_{n}^{e}, I(x) * M_{n}^{o}\right]
$$

Phase congruency can be calculated as [6]

$$
P C(x)=\frac{E(x)}{\sum_{n} A_{n}(x)+\epsilon},
$$

where $\epsilon$ is a small value dependent on the precision of computation [6]. $A_{n}(x)$ represents the sum of amplitudes across all scales

$$
\sum_{n} A_{n}(x) \simeq \sum_{n} \sqrt{e_{n}(x)^{2}+o_{n}(x)^{2}},
$$

and $E(x)$ is the local energy function:

$$
E(x)=\sqrt{F(x)^{2}+H(x)^{2}},
$$

with $F(x)$ and $H(x)$ being the sum of the even and odd filters components across all the scales respectively

$$
\begin{aligned}
& F(x) \simeq \sum_{n} e_{n}(x) \\
& H(x) \simeq \sum_{n} o_{n}(x)
\end{aligned}
$$

Five different frequency bands spanning over $1.5 \mathrm{~Hz}$ to $15.5 \mathrm{~Hz}$ were selected to reject higher frequency electromyography (EMG) activity, with each filter having a bandwidth of one octave. The traditional phase congruency in (2) calculated for an EEG signal of $20 \mathrm{~s}$ duration is shown in Fig. 1(a). A spike has been marked at $10 \mathrm{~s}$ in Fig. 1(a). It can be seen how there is little variation between the phase congruency of the spike and the background EEG. This information has been lost due to the noise introduced by the background EEG data on either side of the interictal spike.

\section{B. Interference in EEG phase congruency computations}

From the point of view of epileptic activity identification using phase congruency calculations, there are two different forms of interference that can obscure the signal of interest, i.e. in this case, interictal activity: normal background electrical activity of the patient's brain and, contamination from movement of cranio-facial muscles. Background electrical activity in a short time window around the interictal manifestation can be clinically useful to the neurologist and hence should not be eliminated. Muscle movements however, could unnecessarily obscure the EEG making these data sections hard to interpret. An estimation of muscle activity incorporated in the phase congruency calculations could be used to compensate the phase congruency calculation when there is significant muscle movement. This could reduce the chances of those sections being wrongly identified to be of diagnostic relevance and hence be transmitted.

\section{Dynamic Noise Compensation}

The most popular technique for denoising phase congruency [6] subtracts the noise threshold from the local energy $E(x)$ in (2). The estimated noise is assumed to be additive and have a constant noise power spectrum. In contradiction, the power spectrum of muscle activity varies during contraction as a function of time [11]. Hence, it could be a priori expected that dynamic estimation of EMG contamination could improve the accuracy of phase congruency calculation.

Although EMG activity has been proved to contaminate scalp EEG signals at all frequencies [11], previous work has shown that there is an increase in power at frequencies above $15 \mathrm{~Hz}$ [11], [12]. [13] also showed that frontal and temporal muscle activity had maximum amplitudes at frequencies ranging from $20 \mathrm{~Hz}$ to $30 \mathrm{~Hz}$ and $40 \mathrm{~Hz}$ to $89 \mathrm{~Hz}$ respectively. In order to estimate the spectral power of muscle activity, the wavelet amplitude $A_{m}(x)$ of the EEG signal was computed using log Gabor wavelet filters spanning $15.5 \mathrm{~Hz}$ to $64 \mathrm{~Hz}$ (scales $m$ ). The phase congruency calculation in (2) is then modified to introduce the higher frequency amplitudes in such a way that the phase congruency is attenuated at 


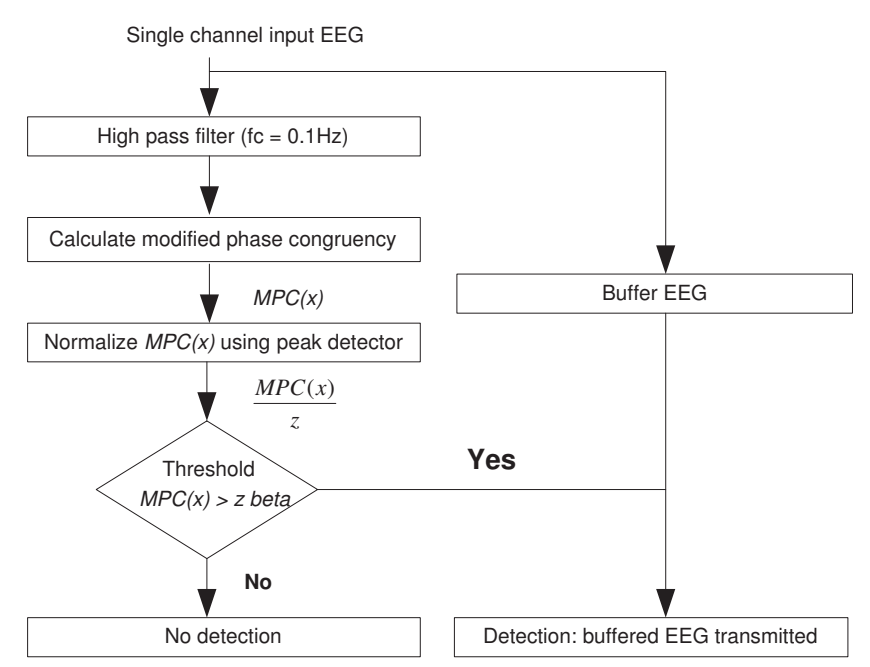

Fig. 2. Data selection algorithm to identify interictal spikes

time $x$ when $A_{m}(x)$ is high:

$$
M P C(x)=\frac{E(x)}{\sum_{n} A_{n}(x)+\epsilon+\sum_{m} A_{m}(x)}
$$

This technique is illustrated in Fig. 1(b), where modified phase congruency $(M P C(x))$ is calculated for $20 \mathrm{~s}$ of EEG data. It can be seen how the expert marked spike at $10 \mathrm{~s}$ has a higher $\operatorname{MPC}(x)$ than the surrounding EEG and can be clearly distinguished using a threshold at 0.6. When compared to traditional phase congruency discussed in Section II-A and calculated for the same $20 \mathrm{~s}$ data in Fig. 1(a), it is clear that the spike is much easier to identify using modified phase congruency.

\section{Proposed data selection algorithm}

The proposed data selection algorithm for identifying interictal spikes is illustrated in Fig. 2. Modified phase congruency is calculated to distinguish between spikes and background EEG. On identifying a spike, a short duration of data before and after the detection (recording window) is transmitted. The duration of the recording window can be chosen to allow background EEG on either side of the detected spike to be transmitted. This additional information could aid neurologists in diagnosing a spike from the transmitted candidate events. Thus, recording windows of $1 \mathrm{~s}, 2.5 \mathrm{~s}, 5 \mathrm{~s}$ and $10 \mathrm{~s}$ have been chosen for performance evaluation in Section III.

With reference to Fig. 2, the algorithm buffers the duration of the recording window from the input single channel EEG. The input EEG data is first high pass filtered at $0.1 \mathrm{~Hz}$ (as recommended in [14]). Modified phase congruency is then calculated as discussed in Section II-C, for each data sample $x$. As interictal spikes are expected to have a higher phase congruency than background EEG, a fixed threshold could be applied to distinguish between spikes and background. Prior to thresholding, modified phase congruency has to be normalized between 0 and 1 . Hence, $M P C(x)$ is passed through a peak detector to store the maximum (or peak) value, $z$. If modified phase congruency at a later time $x+\delta$, exceeds $z$, the $\operatorname{MPC}(x+\delta)$ value is then stored. To normalize modified phase congruency, $M P C(x)$ is divided by $z$. The normalized feature is then compared with a fixed threshold $\beta$. If $M P C(x)>z \beta$, then a detection occurs and the buffered EEG is transmitted. If normalized $M P C(x)$ falls below the threshold, no detection occurs.

\section{E. EEG test database}

The data selection algorithm was tested on 21 EEG data sections across 10 channels: F7, F8, FP1, FP2, O1, O2, T3, T4, T5 and T6. The total duration of the data was over 35 hours. The data contained 899 spike events and corresponds to 13 randomly chosen patients who had been monitored with scalp EEG. The events were marked by expert markers from the National Society of Epilepsy, UK.

\section{PERFORMANCE ANALYSIS}

The percentage sensitivity and data transmitted were calculated to characterize the performance of the algorithm. Sensitivity is generally defined as the number of correctly detected events as a fraction of the number of expert marked events. Following the recommendation in [15] time/event weighted sensitivity is used, in order to reduce dependency of the results on the database. Time/event weighted sensitivity is given as

$$
\text { Sensitivity }=\frac{1}{\sum_{i=1}^{M} \frac{T_{i}}{N_{i}}} \sum_{i=1}^{M} \frac{D_{i}}{N_{i}} \frac{T_{i}}{N_{i}} \times 100 \%
$$

where $D_{i}$ is the number of correctly detected events in a data section $i$, being the total number of datasets analyzed $M$. Each data section has a duration $T_{i}$ and $N_{i}$ expert marked events.

The percentage sensitivity and data transmitted varies with the chosen threshold $\beta$. A receiver operating characteristic (ROC) curve can be plotted by varying $\beta$ from 0 to 1 , as shown in Fig. 3. Each sensitivity and data transmitted value plotted in Fig. 3 corresponds to a different value of $\beta$. When $\beta$ equals zero, every sample is detected and transmitted, giving $100 \%$ sensitivity and data transmitted. For $\beta$ equal to one, there will be very few detections; hence sensitivity and data transmitted will tend to zero. Different values of $\beta$ will give different trade-off points in-between.

\section{A. Baseline performance}

Fig. 3 shows the time/event weighted sensitivity and percentage of data transmitted for the modified phase congruency algorithm. The proposed algorithm has been plotted for four different durations of recording window. The dashed $\mathrm{y}=\mathrm{x}$ line depicts a random or chance detector, as a random detector would achieve $50 \%$ sensitivity when $50 \%$ of data is transmitted by chance.

With reference to Fig. 3, it can be seen that modified phase congruency identifies $90 \%$ of expert marked spikes whilst transmitting almost $45 \%$ of the analyzed data. At this threshold, the $1 \mathrm{~s}$ recording window achieves the lowest 


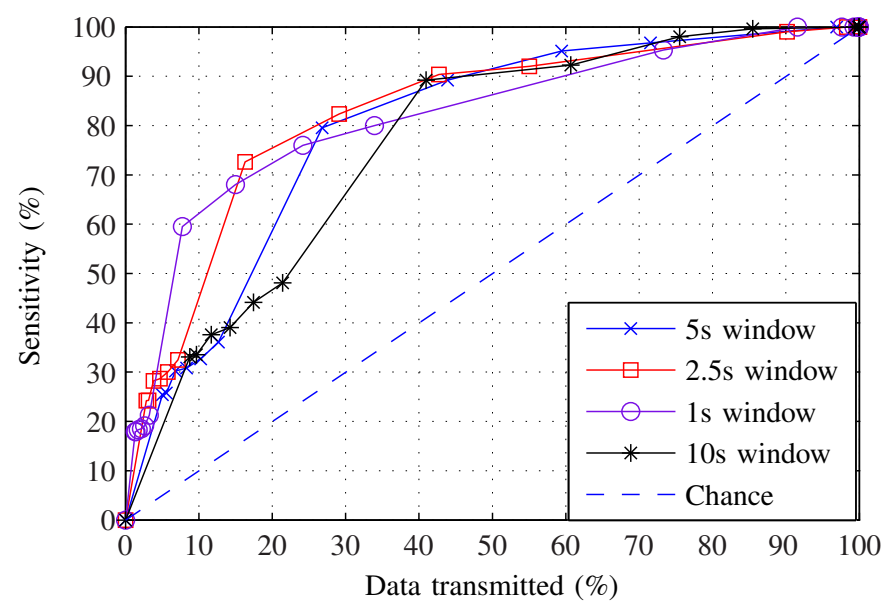

Fig. 3. Performance of modified phase congruency algorithm for different recording windows. Chance performance is depicted as $\mathrm{y}=\mathrm{x}$ line

TABLE I

PERFORMANCE OF THREE DIFFERENT PHASE CONGRUENCY METHODS

\begin{tabular}{|c|ccc|}
\hline Time/event & \multicolumn{3}{|c|}{ Data reduction } \\
\cline { 2 - 4 } $\begin{array}{c}\text { weighted } \\
\text { sensitivity }\end{array}$ & $\begin{array}{c}\text { Traditional phase } \\
\text { congruency }\end{array}$ & $\begin{array}{c}\text { Denoised phase } \\
\text { congruency }\end{array}$ & $\begin{array}{c}\text { Proposed } \\
\text { algorithm }\end{array}$ \\
\hline $50 \%$ & $50 \%$ & $85 \%$ & $75 \%$ \\
\hline $80 \%$ & $35 \%$ & $60 \%$ & $65 \%$ \\
\hline $90 \%$ & $30 \%$ & $40 \%$ & $55 \%$ \\
\hline
\end{tabular}

data reduction of $40 \%$, while the other recording windows achieve $>55 \%$ data reduction. All four recording windows have similar performance at low sensitivity, with a maximum difference of $20 \%$ data transmitted at $60 \%$ sensitivity. The implemented algorithm performs significantly better than a chance detector, at all values of $\beta$.

\section{B. Comparison with alternative methods}

Traditional phase congruency as described in (2) and the most cited denoised phase congruency [6] have been tested on different applications, including seizure detection in epileptic EEG [16]. Hence the performance of the proposed algorithm is compared with these two methods, by characterizing the sensitivity and data transmitted of all three methods on the same EEG database (see Table I). The data reduction achieved by the top three recording windows have been included in Table I, for 50\%, $80 \%$ and $90 \%$ time/event weighted sensitivity.

From Table I, it can be seen that traditional phase congruency has the lowest data reduction at all values of sensitivity. At 50\% sensitivity, denoised phase congruency has the highest data reduction, but performs worse than modified phase congruency at higher sensitivity values. At a nominal $90 \%$ sensitivity, modified phase congruency transmits $15 \%$ less data than denoised phase congruency.

\section{CONClusions And Future Work}

A data selection algorithm to identify interictal spikes in scalp EEG data has been proposed. This proposed algorithm computes phase congruency and incorporates an estimation of muscle artefacts to reduce interference in the calculated phase congruency value. This modified phase congruency algorithm identified $90 \%$ of interictal spikes whilst transmitting only $45 \%$ of EEG data. At $90 \%$ sensitivity, the data reduction achieved by this algorithm is $25 \%$ more than traditional phase congruency and $15 \%$ more than the state-ofthe-art denoised phase congruency when tested on the same database.

So far, only sensitivity and data reduction have been evaluated for the proposed algorithm. For a data reduction algorithm to be beneficial in a wireless ambulatory EEG system, the power consumption for implementing the algorithm in hardware should be less than the power saving from reduced transmission [17]. Therefore, further work is needed to quantify the power consumption of this data selection algorithm. Furthermore, an algorithm to select epileptic seizures should be developed and implemented in parallel to this data selection algorithm, in order to record all epileptiform activity.

\section{REFERENCES}

[1] S. Sisodiya, "Etiology and management of refractory epilepsies," Nat. Clin. Pract. Neuro., vol. 3, no. 6, pp. 320-330, 2007.

[2] E. Waterhouse, "New horizons in ambulatory electroencephalography," IEEE Eng. Med. Biol. Mag., vol. 22, no. 3, pp. 74-80, 2003.

[3] R. P. Lesser, "Epilepsy: Does continuous EEG monitoring improve seizure control?" Nat. Rev. Neurol., vol. 5, no. 11, pp. 581-582, 2009.

[4] A. J. Casson, S. Smith, J. S. Duncan, and E. Rodriguez-Villegas, "Wearable EEG: what is it, why is it needed and what does it entail?" in IEEE EMBC, 2008, pp. 5867-5870.

[5] R. F. Yazicioglu, P. Merken, R. Puers, and C. Van Hoof, "A $60 \mu \mathrm{W}$ $60 \mathrm{nV} / \sqrt{\mathrm{Hz}}$ readout front-end for portable biopotential acquisition systems," IEEE J. Solid-State Circuits, vol. 42, no. 5, pp. 1100-1110, 2007.

[6] P. Kovesi, "Image features from phase congruency," Videre: J. Computer Vision Research, vol. 1, no. 3, 1999.

[7] H. Zhi-Kai, L. De-Hui, Z. Wei-Zhong, and H. Ling-Ying, "Multi-view face database recognition using phase congruency and SVM classifier,' in ICCEE, Phuket, December 2008, pp. 219-222.

[8] Y. Punsawad and Y. Wongsawat, "Palmprint image enhancement using phase congruency," in IEEE ROBIO, Bangkok, February 2009, pp. 1643-1646.

[9] S. Venkatesh and R. Owens, "An energy feature detection scheme," in Int. Conf. Image Processing, 1989, pp. 553-557.

[10] J. Gotman, "Automatic detection of seizures and spikes," J. Clin. Neurophysiol., vol. 16, no. 2, pp. 130-140, 1999.

[11] R. D. O'Donnell, J. Berkhout, and W. R. Adey, "Contamination of scalp EEG spectrum during contraction of cranio-facial muscles," Electroencephalogr. and Clin. Neurophysiol., vol. 37, no. 2, pp. 145$151,1974$.

[12] J. Gotman, J. R. Ives, and P. Gloor, "Frequency content of EEG and EMG at seizure onset:possibility of removal of EMG artefact by digital filtering," Electroencephalogr. and Clin. Neurophysiol., vol. 52, pp. 626-639, 1981.

[13] I. I. Goncharova, D. J. McFarland, T. M. Vaughan, and J. R. Wolpaw, "EMG contamination of EEG: spectral and topographical characteristics," Clin. Neurophysiol., vol. 114, no. 9, pp. 1580-1593, 2003.

[14] M. R. Nuwer, G. Comi, R. Emerson, A. Fuglsang-Frederiksen, J.-M. Gurit, H. Hinrichs, A. Ikeda, F. Jose C. Luccas, and P. Rappelsburger, "IFCN standards for digital recording of clinical EEG," Electroencephalogr. and Clin. Neurophysiol., vol. 106, no. 3, pp. 259-261, 1998.

[15] A. J. Casson and E. Rodriguez-Villegas, "Towards online data reduction for portable electroencephalography systems in epilepsy," IEEE Trans. Biomed. Eng., vol. 56, no. 12, pp. 2816-2825, 2009.

[16] Y. Wongsawat, "Epileptic seizure detection in EEG recordings using phase congruency," in IEEE EMBC, Vancouver, August 2008, pp. 927930.

[17] A. J. Casson and E. Rodriguez-Villegas, "Generic vs custom; analogue vs digital: on the implementation of an online EEG signal processing algorithm," in IEEE EMBC, Vancouver, August 2008, pp. 5876 - 5880. 